\title{
Optical Study of ZnTe-Based 2D and 0D Photonic Structures Containing CdTe/ZnTe Quantum Dots
}

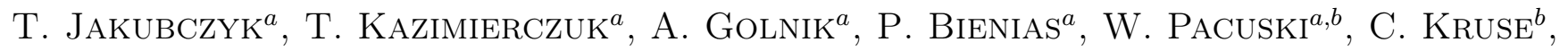 \\ D. Hommel ${ }^{b}$, Ł. KŁopotowski ${ }^{c}$, T. Wojtowicz ${ }^{c}$ AND J.A. GAJ ${ }^{a}$ \\ ${ }^{a}$ Institute of Experimental Physics, University of Warsaw, Hoża 69, 00-681 Warsaw, Poland \\ ${ }^{b}$ Institute of Solid State Physics, University of Bremen, Postfach 330 440, D-28334 Bremen, Germany \\ ${ }^{c}$ Institute of Physics, Polish Academy of Sciences, al. Lotników 32/46, 02-668 Warsaw, Poland
}

We report on an optical study of ZnTe-based microcavity and micropillars. Angle-resolved reflectivity studies confirm a high quality of the investigated structure by setting the lower bound on the quality factor $Q \geq 1000$, determined from normal-incidence reflection spectra. In a microphotoluminescence study, micropillar modes are observed at temperatures of the order of tens of kelvins. For structures grown by a complex growth procedure at two different MBE facilities, an enhancement of photoluminescence in the cavity mode is observed.

PACS numbers: 78.67.Pt

\section{Introduction}

An important progress has been recently achieved in fabrication of telluride-based II-VI photonic structures. Distributed Bragg reflectors (DBRs) were grown by the molecular beam epitaxy (MBE), using a new low refractive index composite material — a short-period superlattice based on ZnTe, MgTe, and MgSe binary compounds [1]. A high refractive index contrast $\Delta n=0.5$ was thus achieved, resulting in a wide $(60 \mathrm{~nm})$ stop band with high $(>0.99)$ reflectivity.

In this work, we present optical studies of CdTe quantum dots (QDs), embedded in photonic structures based on these new DBRs. The quantum dots were incorporated in photonic structures of two types: microcavities (2D photon confinement) and microcavity-based micropillars (0D photon confinement). The micropillars, of diameters $1,2,3$, and $5 \mu \mathrm{m}$, presented schematically in Fig. 1, were fabricated using a focused ion beam (FIB) facility.

\section{Results}

Photonic 2D confinement was characterized by reflectivity measurements performed on microcavities, yielding the resonance energy and the quality factor in each case. Reflectivity spectra (see Fig. 2) reveal a cavity mode around $1915 \mathrm{meV}$ for normal light incidence. The mode width is equal to about $2 \mathrm{meV}$, yielding a quality factor $Q \approx 1000$, an evidence of high quality (good lattice match of the layers and low dislocation density) of the structure obtained. This value is only the lower bound on the $Q$ value because of a lateral inhomogeneity of the structure (the mode energy shifts by $2 \mathrm{meV}$ per $1 \mathrm{~mm}$ displacement). A finite size of the light spot (several hundreds of $\mu \mathrm{m}$ ) results in a broadening of the

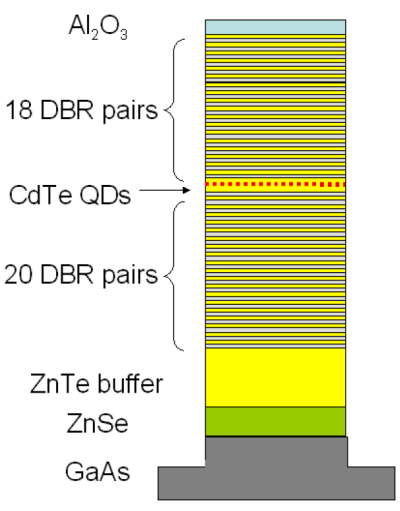

Fig. 1. Scheme of a micropillar etched out of a microcavity. The structure is designed to have all the layers above the ZnTe buffer lattice-matched to ZnTe.

cavity mode. The reflectivity spectra have been well reproduced by simulations based on the material data and structure parameters, as shown in Fig. 2. A shift of the cavity mode, accompanied by a decrease of the $Q$ value, observed at higher incidence angles agrees with the simulation results. Rough estimation of the light spot size to have a diameter of $0.2 \mathrm{~mm}$ gives a $10 \%$ thickening of the FWHM of the cavity mode if its width is equal to $2 \mathrm{meV}$.

0D photonic modes in micropillars were revealed in microphotoluminescence (microPL) spectra measured at several tens of kelvins. At lower temperatures, single QD lines were observed, while at higher temperatures the individual QD lines disappeared and a characteristic pattern, dependent on micropillar size, revealed eigenmodes due to the 0D photonic confinement (see Fig. 3). The spectrum measured at $65 \mathrm{~K}$ presents well separated eigenmodes of the pillars. At this temperature, the individual QD lines broaden due to the interaction with 


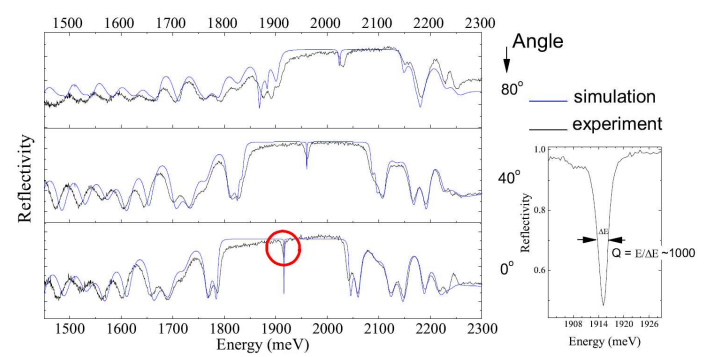

Fig. 2. Experimental and simulated reflectivity spectra of a microcavity for three different angles of incidence. High resolution spectrum of the cavity mode (inset) reveals a lower bound for the value of the cavity mode $Q \geq 1000$.

phonons and merge into a continuous background, which allows the cavity modes to appear in the spectrum [2]. These modes were observed for all the micropillars with diameters down to $1 \mu \mathrm{m}$, proving a high precision of the FIB processing.

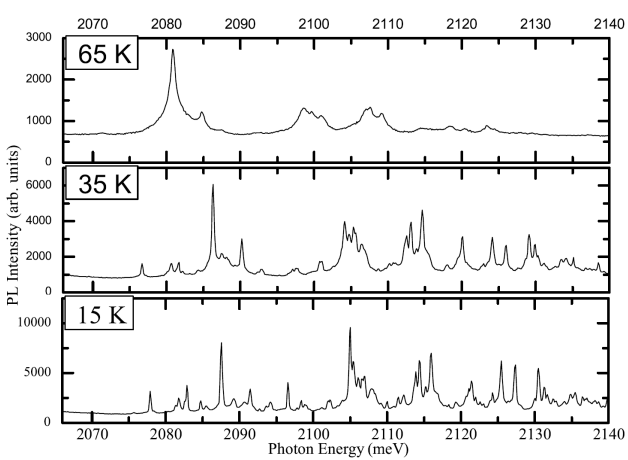

Fig. 3. MicroPL of $1 \mu \mathrm{m}$ diameter micropillar under high excitation power and at different temperatures. Individual QD lines gradually disappear with temperature growth and a characteristic pattern, dependent on micropillar size, gradually reveals the micropillar eigenmodes due to 0D photonic confinement.

\section{Discussion}

The results presented so far were obtained on samples grown entirely at University of Bremen. In addition, some samples were grown at two different MBE facilities - the photonic structures at University of Bremen and quantum dots at Institute of Physics of Polish Academy of Sciences. The sample structure was protected with a tellurium layer during the necessary transfers before and after the QD growth. MicroPL experiments show that this growth procedure allowed us to incorporate successfully a self-assembled QD plane in a microcavity. Sharp lines of individual QDs were observed at liquid helium temperature and low excitation power for a microcavity containing QDs, as well as for a reference sample without the photonic structure. Although the procedure requires further improvement (e.g. increase of the quality factor), the enhancement of the emission lines of single QDs by the cavity mode is clearly visible in Fig. 4, where the microPL spectrum is compared to the reflectivity spectrum.

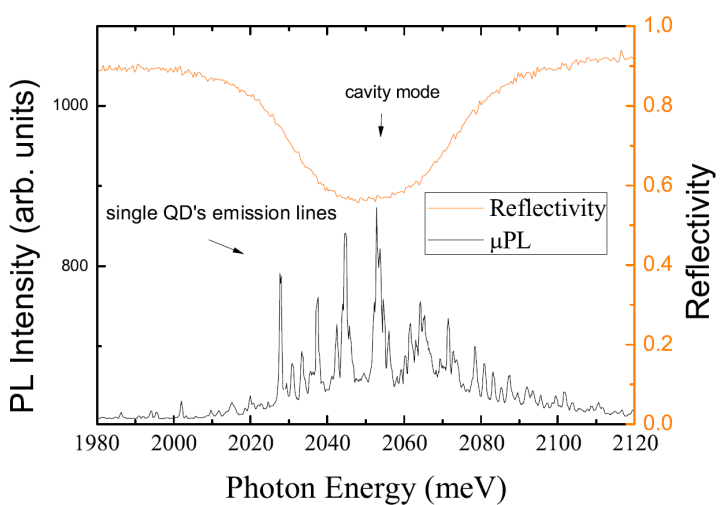

Fig. 4. Reflectivity and microPL spectra revealing enhancement of emission in the cavity mode for a structure grown at two different MBE facilities.

\section{Conclusion}

In conclusion, our optical study confirmed a high quality of the recently developed ZnTe-based Bragg reflectors and their applicability for preparation of photonic structures. High quality is confirmed by high $Q$ observed (at least 1000). This sets a promising perspective of placing individual QDs containing single magnetic Mn ions in $[3-5]$ in such structures and controlling the spontaneous emission of selected emission lines of such QDs.

\section{Acknowledgments}

This work was supported by Polish Ministry of Science and Higher Education (contract financed in 2007-2010), Marie Curie Actions (contract number MTKD-CT-2005-029671), and Alexander von Humboldt Foundation. We thank Steve Byrnes for making available his simulation program.

\section{References}

[1] W. Pacuski, C. Kruse, S. Figge, D. Hommel, Appl. Phys. Lett. 94, 191108 (2009).

[2] K. Sebald, C. Kruse, J. Wiersig, Phys. Status Solidi B 241, 731 (2004).

[3] L. Besombes, Y. Léger, L. Maingault, D. Ferrand, H. Mariette, J. Cibert, Phys. Rev. Lett. 93, 207403 (2004).

[4] M. Goryca, T. Kazimierczuk, M. Nawrocki, A. Golnik, J.A. Gaj, P. Kossacki, P. Wojnar, G. Karczewski, Phys. Rev. Lett. 103, 087401 (2009).

[5] P. Wojnar, G. Karczewski, M. Aleszkiewicz, J. Suffczyński, K. Kowalik, A. Golnik, J. Kossut, J. Phys. Conf. Ser. 146, 012032 (2009). 